\title{
LA-UR- $94-345$
}

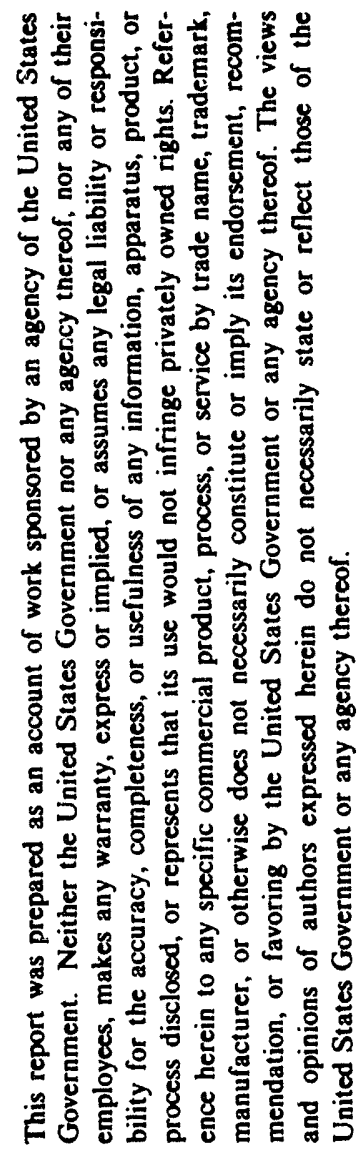

Author(s):

D. B. Harris, and R.E. Chrien

\section{Submitted to:}

Laser Interaction and Related Plasma Phenomena Monterey, CA

October 1993

\section{Los Alamos}

NATIONAL LABORATORY

Los Alamos National Laboratory, an affirmative action/equal opportunity employer, is operated by the University of California for the U.S. Department of Energy under contract W.7405-ENG-36. By acceptance of this article, the publisher recognizes that the U.S. Government retains a nonexclusive, royalty-free license to publish or reproduce the published form of this contribution, or to allow others to do so, for U.S. Government purposes. The Los Alamos National Laboratory

requests that the publisher identity this article as work performed under the auspices of the U.S. Department of Energy.

dt 


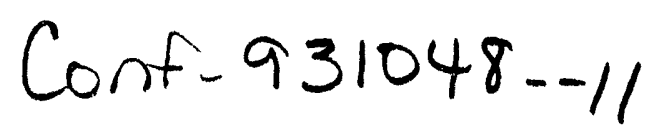

\title{
MEASUREMENT OF ICF FUEL ION TEMPERATURE ON NOVA*
}

\author{
David B. Harris and Robert E. Chrien \\ Los Alamos National Laboratory \\ Mail Stop F645 \\ Los Alamos, New Mexico 87545
}

\begin{abstract}
A new diagnostic for measuring the average fuel ion temperature in inertial confinement fusion (ICF) targets at the time of peak burn has been constructed by Los Alamos and installed on the Nova Laser System at Lawrence Livermore National Laboratory. This ion-temperature diagnostic measures the time-of-flight of fusion neutrons and determines the thermonuclearreaction-weighted ion temperature through the time-of-arrival distribution. Preliminary experiments have been designed and performed to test the diagnostic. These tests measured the ion temperature of targets designed to have varying temperatures and yields. Additionally, an experiment has been designed to examine the cause of the increase in yield degradation (compared to clean 1-D calculations) as the capsule convergence is increased. Understanding the cause of yield degradation in high convergence implosions is necessary to increase the confidence of the target performance for the next generation National Ignition Facility planned by the US ICF Program.
\end{abstract}

\section{INTRODUCTION}

Diagnostics for inertial confinement fusion (ICF) targets are very difficult because of the short time scales (nanoseconds for the laser pulse and tens of picoseconds for the fusion burn duration in current ICF facilities), yet diagnostics are crucial for determining target performance. The Nova Laser at Lawrence Livermore National Laboratory (LLNL) has stateof-the-art diagnostics, developed primarily by LLNL. Los Alamos National Laboratory is working closely with LLNL on Nova target physics experiments and has developed and fielded several important diagnostics, primarily in the area of $x$-ray imaging. A new diagnostic, measuring the fusion-product neutron time-of-flight, has recently been developed and installed by Los Alamos on the Nova Laser.

This new diagnostic, known as Tion ("tee-ion"), can determine the fusion-reaction-rateweighted ion temperature, which is closely approximated by the ion temperature at the time of peak burn weighted by the radial distribution of the number of fusion reactions. Targets have been designed and fielded to test the response of the Tion diagnostic to varying target temperatures and yields. Additionally, some direct-drive targets with $1 \%$ tritium and $99 \%$ deuterium have been shot to experimentally determine the instrument response time.

* This work supported by the US Department of Energy. 
The new capability to determine the fuel ion temperature has led to the design of an experiment to investigate the cause of increased yield degradation (compared to clean 1-D calculations) as the convergence ratio of the target implosion is increased. The phenomena of yield degradation has been observed in both indirect drive ${ }^{(1)}$ and direct drive ${ }^{(2)}$, and is a major concern for ignition or high-gain ICF facilities proposed for the future. This yield degradation is most likely caused by mix and/or asymmetry. The Nova high-convergence target experiment to investigate the cause of yield degradation is being performed as part of the Nova experiments designed to validate the physics for the proposed next-generation indirect-drive ICF facility, called the National Ignition Facility ${ }^{(3)}$.

\section{DESCRIPTION OF THE TION DETECTOR}

The Tion neutron diagnostic measures the neutron arrival-time distribution using an array of 1020 scintillator-photomultiplier detectors with about 1-ris time resolution and operates in the single-hit mode ${ }^{(4)}$. The ion temperature, $T_{i}$ is determined from the FWHM neutron energy spread $\Delta \mathrm{E}_{\mathrm{n}}$ according to the relation ${ }^{(5)}$

$$
\Delta \mathrm{E}_{\mathrm{n}}=\mathrm{C}_{\mathrm{dd}} \mathrm{T}_{\mathrm{i}}^{1 / 2}
$$

where $\mathrm{T}_{\mathrm{i}}$ and $\Delta \mathrm{E}_{\mathrm{n}}$ are both in $\mathrm{keV}$, and $\mathrm{C}_{\mathrm{dd}}=82.5$ (for $\mathrm{D}-\mathrm{T}$ reactions $\mathrm{C}_{\mathrm{dt}}=177$ ). The energy spread is related to the arrival-time spread $\Delta t$ by the relation

$$
\Delta \mathrm{t} / \mathrm{t} \sim-(1 / 2) \Delta \mathrm{E}_{\mathrm{n}} / \mathrm{E}_{\mathrm{n}}
$$

Tion is located $27.10 \mathrm{~m}$ from the center of the Nova target chamber, so a D-D neutron has a time-of-flight of $1254 \mathrm{~ns}$ and $\Delta \mathrm{t}$ for $\mathrm{T}_{\mathrm{i}}=1 \mathrm{keV}$ is $21.7 \mathrm{~ns}$. Tion can cover $\mathrm{D}$-D neutron yields from $\sim 10^{7}$ to $2 \times 10^{9}$, a dynamic range of $\sim 200$. This range is obtained in part by varying the number of channel hits in the array (from 50 to 500), and also by changing the scintillator volume (from 0.2 to $3.6 \mathrm{~cm}^{3}$ ).

The detectors are enclosed in a cylindrical steel chamber that provides magnetic shielding for the photomultiplier tubes (PMTs), as illustrated in Fig. 1. The 1020 PMTs are supported by fore- and back-planes made of black nylon to suppress neutron backscattering and stray light. Each PMT is pressed against a scintillator by a spring behind its base. A silicone pad is used to improve the optical coupling. Additional information on the details of the Tion detector can be found in Reference 6 .

\section{DIAGNOSTIC CHECKOUT EXPERIMENTS}

Initial tests with Tion have characterized the detector time response in the Nova installation. The phototube array, without the scintillators, has been illuminated with 5-ns 337$\mathrm{nm}$ light pulses from a nitrogen laser to correct for differences in propagation time among the channels. These tests indicate a time resolution of about $1.5 \mathrm{~ns}$. In addition, we plan to further characterize the array using direct-drive glass microballoon targets filled with $1 \%$ tritium and $99 \%$ deuterium. The fill was chosen to produce roughly equal D-D and D-T neutron signals in the Tion array. The time spread of the D-T neutrons is more strongly influenced by the detector time response than the time spread of the D-D neutrons, which is dominated by the temperature-induced energy spread. Thus these targets can be used to determine the detector time response. 
(a)

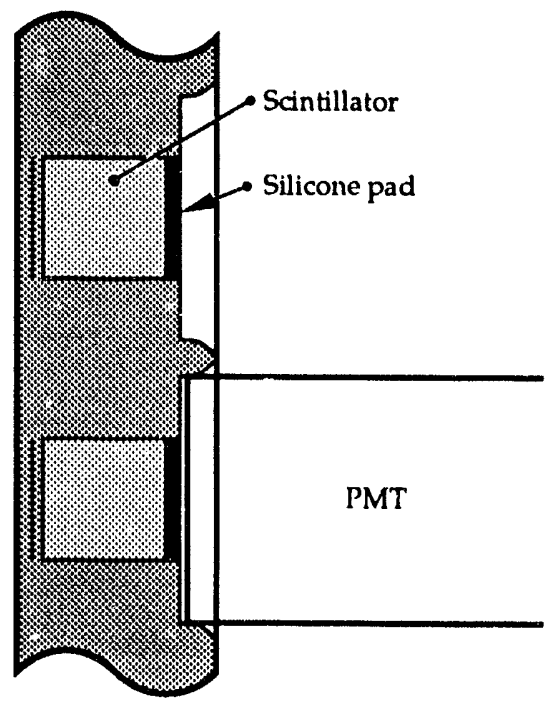

(b)

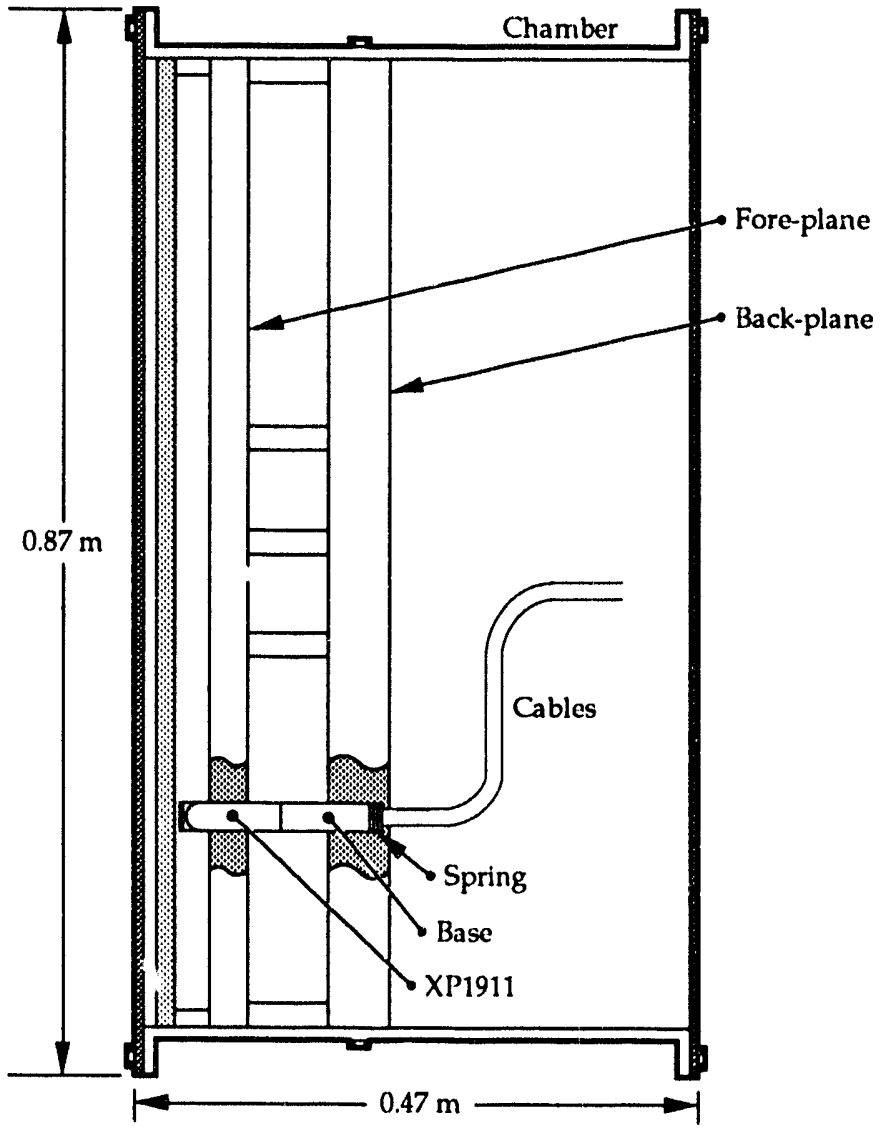

Figure 1. Assembly drawing of the photomultiplier tube (PMT) chamber, showing scintillators, silicone coupling pads, PMTs, bases, and cables for the PMT array.

We have also fielded target experiments designed to test Tion by imploding capsules designed to have significantly different ion temperatures with the same yield. As compared to the low-ion-temperature "standard" capsule, the high-ion-temperature implosions use a lower fill pressure and a thinner capsule wall. LASNEX calculations of these targets predict that the lower-fill pressure, thinner-walled capsule will have a higher temperature by $\sim 1 \mathrm{keV}$ than the standard capsule, with both capsules having a yield of $\sim 1.0 \times 10^{9}$. The experimentally measured neutron time-of-arrival distribution for both of these targets is shown in Fig. 2. As shown, the ion temperature and yield of the standard target was measured to be $1.6 \mathrm{keV}$ and $1.4 \times 10^{9}$, and the lower-fill-pressure, thinner-walled capsule measured ion temperature and yield was $3.6 \mathrm{keV}$ and $9.7 \times 10^{8}$.

\section{HIGH CONVERGENCE EXPERIMENTS}

Experiments to investigate yield degradation (as compared to unmixed 1-D calculated performance) are just beginning. The most likely causes of yield degradation in highconvergence targets are mix and/or asymmetry. The high-convergence target experiments planned have been designed such that the new Tion diagnostic capability and the extensive suite of diagnostics that currently exist on Nova can differentiate between these two degradation mechanisms. All of these planned experiments will be indirectly driven targets. 

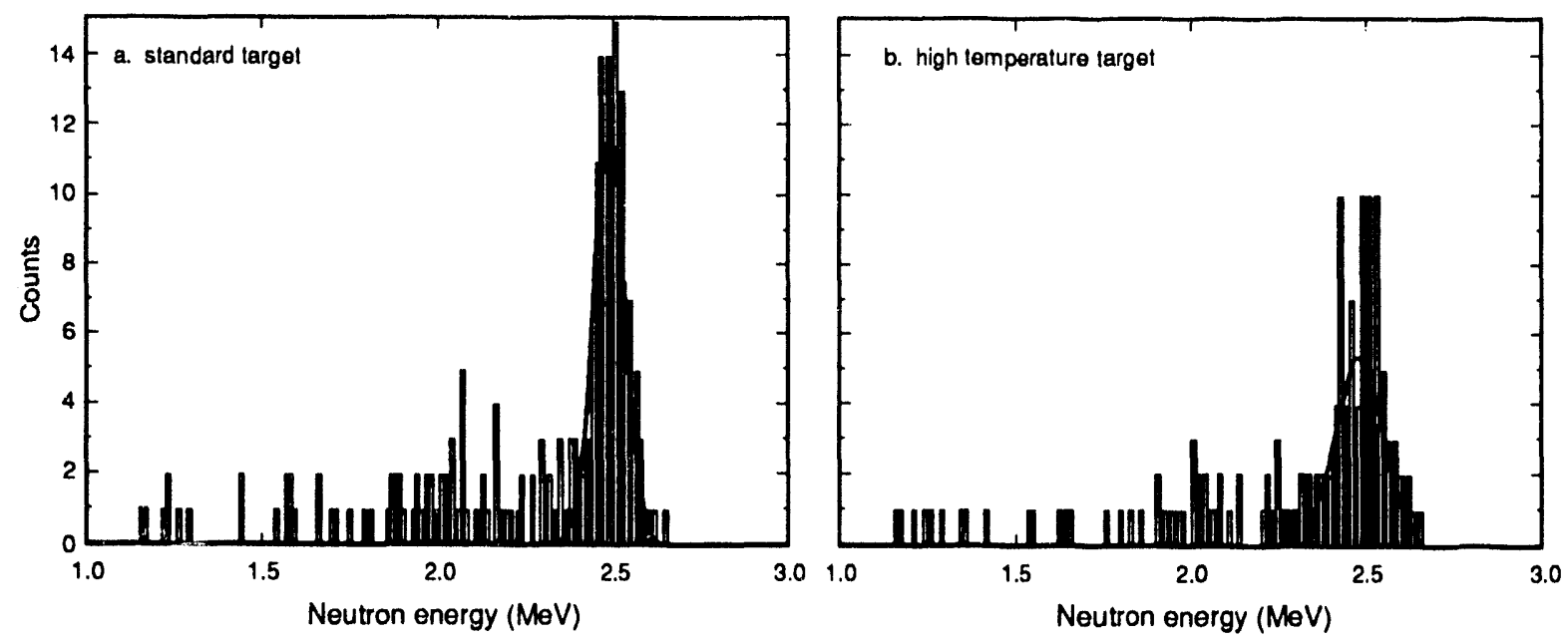

Figure 2. Experimentally measured D-D neutron arrival time distributions for a) a standard target, and b) a target designed to have equal yield as the standard target but higher ion temperature (achieved by lowering the fill pressure and reducing the capsule wall thickness). Measured ion temperatures for these two experiments were a) $1.6 \mathrm{keV}$ and b) $3.6 \mathrm{keV}$.

The high-convergence experiment is designed to use four different targets that gradually increase in convergence ratio. LASNEX calculations of these four targets are shown in Table 1. The series of targets are identical with the exception that the $\mathrm{D}_{2}$ fill pressure will be varied to change the capsule convergence (decreasing the $D_{2}$ fill pressure increases the capsule convergence). All of the targets will have a small amount of argon gas mixed in with the deuterium and a small amount of chlorine in the layer of the target closest to the gas. The argon and chlorine are included to increase the $x$-ray signal from the gas, provide spectroscopic signals for measurement of the gas density and temperature, and to provide a measure of mix (by examining the ratio of the strength of the argon to chlorine lines--brighter $\mathrm{Cl}$ lines in comparison to the Ar lines signifies mix of the pusher into the gas region at the time of peak comression $\left.^{(7)}\right)$.

Table 1. Results of clean 1-D LASNEX calculations of the targets to be used in the highconvergence experiments investigating yield degradation.

\begin{tabular}{|c|c|c|c|c|c|c|}
\hline Target & $\begin{array}{c}\text { Wall } \\
\text { Thickness } \\
(\mu \mathrm{m})\end{array}$ & $\begin{array}{c}\mathrm{D}_{2} \\
\text { Pressure } \\
(\text { arbitrary })\end{array}$ & $\begin{array}{c}\text { Peak Ion } \\
\text { Temperature } \\
(\mathrm{keV})\end{array}$ & $\begin{array}{c}\text { Peak } \mathrm{D}_{2} \\
\rho \mathrm{fr} \\
\left(\mathrm{mg} / \mathrm{cm}^{2}\right)\end{array}$ & $\begin{array}{c}\text { Peak } \mathrm{D}_{2} \\
\text { Density } \\
\left(\mathrm{g} / \mathrm{cm}^{3}\right)\end{array}$ & $\begin{array}{c}\text { D-D } \\
\text { Neutron } \\
\text { Yield } \\
\left(10^{8}\right)\end{array}$ \\
\hline 1 & 55 & 50 & 1.1 & 7.4 & 2.4 & 12.8 \\
\hline 2 & 55 & 10 & 1.4 & 7.8 & 5.3 & 4.2 \\
\hline 3 & 55 & 6 & 1.5 & 8.7 & 7.5 & 3.5 \\
\hline 4 & 55 & 2 & 2.2 & 9.6 & 12.8 & 1.9 \\
\hline
\end{tabular}

Similarly, LASNEX calculations with mix have been performed to determine how mix modifies the yield, ion temperature, and calculated $x$-ray signal. The qualitative result of the LASNEX calculations is that as mix is increased: 
- the target yield decreases because some of the colder pusher material mixes into the deuterium fuel and lowers the ion temperature,

- the reaction-weighted ion temperature increases because the mixing region primarily cools the cooler outer fuel region, so the resulting yield increasingly comes from the hotter center part of the fuel region (note, the mass-averaged fuel region temperature decreases in this case), and

- the x-ray image gets larger and weaker because some of the hot fuel (with a small amount of Argon to increase the x-ray signal) spreads out into the cold pusher material.

The affect of asymmetry has also been calculated using LASNEX. Here, the impact of increasing asymmetry on the yield, ion temperature, and x-ray signal is calculated to show that:

- the target yield decreases as the asymmetry affect is increased

- the reaction-weighted ion temperature decreases slightly due to cooler temperatures throughout the fuel region and lower ion temperatures in the center due to the converging shock being asymmetric, and

- the $x$-ray image is slightly weaker and clearly shows an asymmetric shape.

The results of these calculations are shown in Fig. 3.

The experimental plan for these experiments is to proceed from the lowest convergence target to the highest. For each target design, the following quantities will be measured:

- neutron yield,

- bang time (time of peak neutron emission),

- neutron-weighted ion temperature,

- fuel region $\rho R$ (density-radius product)

- symmetry of the implosion (measuring the emission of $x$-rays from the Ar in the gas region of the target at the time of peak compression)(8),

- mix, via the relative strengths of the $\mathrm{Ar}$ and $\mathrm{Cl}$ lines ${ }^{(7)}$, and

- density and temperature of the gas region at peak compression via spectroscopic techniques ${ }^{(9)}$.

These experiments are scheduled to begin in December, 1993.
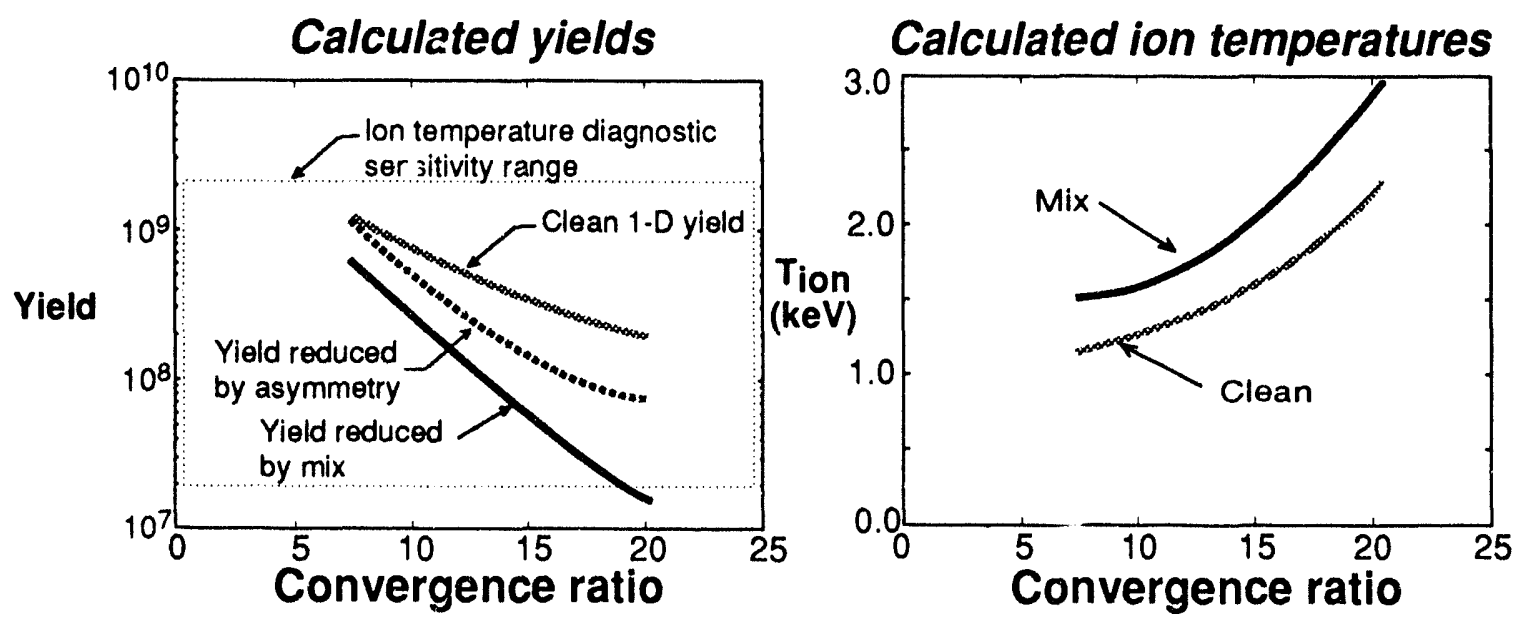

Figure 3. The result of LASNEX calculations for the high convergence targets, a) Calculated yield as a function of convergence ratio for mix degradation and degradation due to asymmetry. b) Calculated ion temperature as a function of convergence ratio for clean 1-D and mixed targets. 


\section{SUMMARY}

A new diagnostic, called Tion ("tee-ion"), has been constructed and installed on the Nova Laser at Lawrence Livermore National Laboratory. This new diagnostic measures the fuel ion temperature inside the core of an imploded ICF target at the time of peak reaction rate. The ion temperature is determined by measuring the time-of-flight distribution of fusion neutrons escaping the target. Experiments have been performed to test Tion by imploding targets calculated to have varying ion temperatures. Targets were shot on Nova for two different yields to test Tion over a range of target yields.

An experiment has been designed to examine the cause of yield degradation as the convergence ratio of the target is increased. This experiment is now possible because of the new capability to measure the ion temperature using Tion. Asymmetry and/or mix are the two most likely candidates for yield degradation. The cause of the yield degradation is expected to be identified by this experiment using Tion and other existing diagnostics on Nova.

\section{ACKNOWLEDGMENTS}

The authors would like to acknowledge the contributions of M. Cable and M. Nelson in the design of the Tion detector and in the planned experiments (in addition to many others at LLNL and Los Alamos for their constructive comments during various presentations and the design review). The authors would also like to thank D. Simmons and D. Holmberg of EG\&G Energy Measurements in Las Vegas for their contributions in the construction of Tion. Finally, the authors appreciate the editing assistance of Pat Medius and the illustration support of Maida Trujillo.

\section{REFERENCES}

1. J. D. Lindl, "Progress on Achieving the ICF Conditions Needed for High Gain," Fusion Technology, 15, 227 (1989).

2. $\quad$ "Laser-Fusion Target Implosion Studies with Omega," LLE Review, 28, 155 (1986).

3. Second Review of the Department of Energy's Inertial Confinement Fusion Program Final Report, National Academy Press, Washington (1990).

4. M. D. Cable, "Inertial Confinement Fusion Ion Temperature Measurements using a Single-Hit Detector Array," J. Appl. Phys. 60, 3068 (1986).

5. H. Brysk, "Fusion Neutron Energies and Spectra," Plasma Physics, 15, 611 (1973).

6. R. E. Chrien, D. B. Harris, M. D. Cable, M. B. Nelson, D. F. Simmons, and D. L. Holmberg, "Neutron Time-of-Flight Ion Temperature Diagnostic for Nova," Lawrence Livermore National Laboratory ICF Quarterly Report, 3, 2, 97 (1993).

7. C. J. Keane et al., "Design of Spectral Diagnostics of Mix in High Growth Factor Nova Implosions," Bulletin of the American Physical Society, 38, 2043 (1993).

8. A. A. Hauer, et al., "Review of Drive Symmetry Measurement and Control Experiments on the Nova Laser System," Bulletin of the American Physical Society, 38, (1993).

9. G. D. Pollak, N. D. Delamater, and J. K. Nash, "Development of Non-LTE Spectral Post-Processor for Dense Plasma Simulations with Application to Spectroscopic Diagnostics in Spherical Implosions at Nova," J. Quantitative Spectroscopy and Radiation Transport, to be published, November, 1993. 

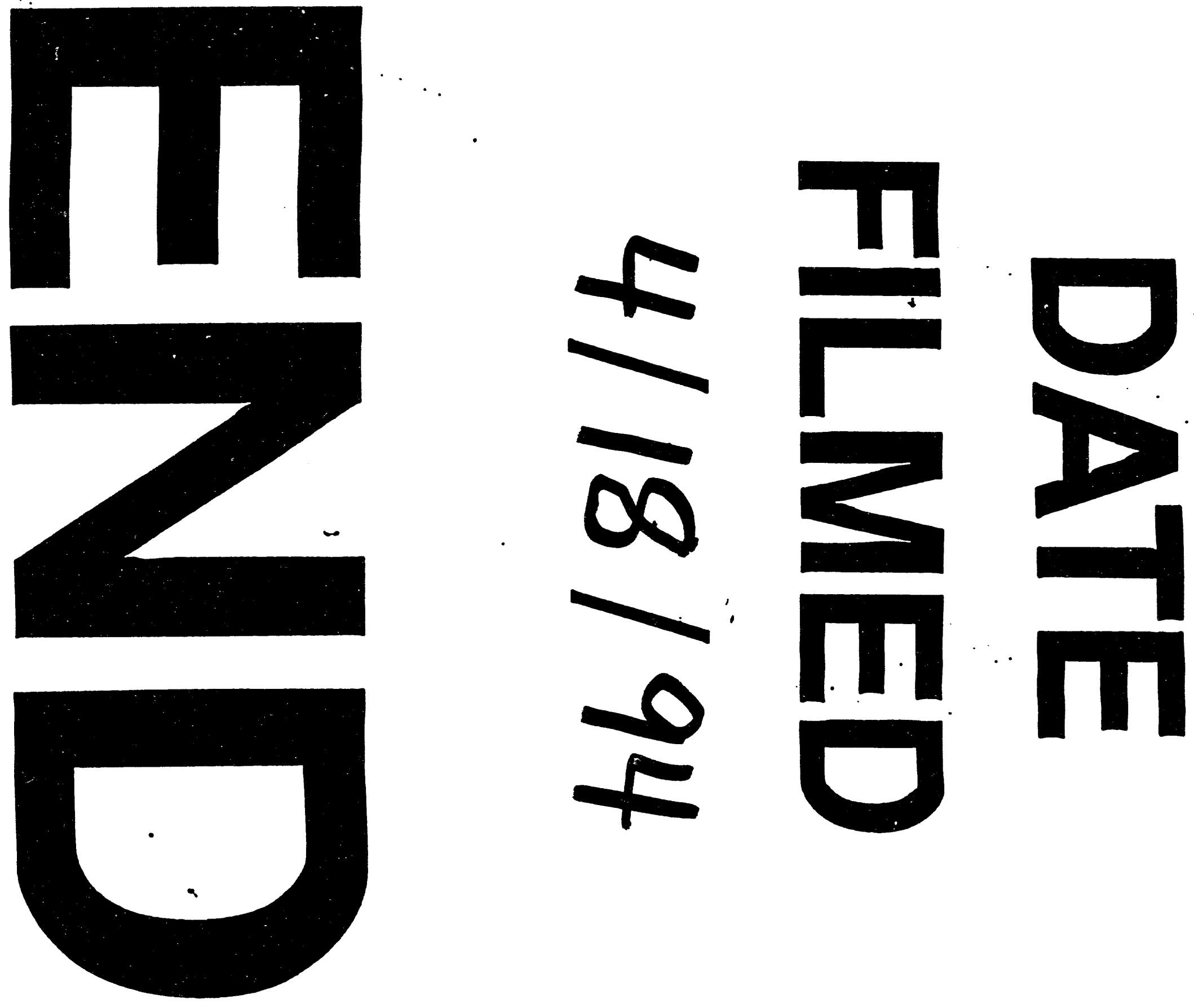
